\title{
Fabrication of an Implantable Micro-pressure Sensor to Measure Deviation Within the Cochlea
}

\author{
Fabricación de un Micro Sensor de Presión Implantable \\ Aplicado a Medir Desviaciones Dentro de la Cochlea
}

\author{
Perez Leonardo ${ }^{1}$, Puers Robert ${ }^{2}$, Volckaerts Bart ${ }^{3}$ \\ ${ }^{1}$ Departamento de electrotecnica - ESAT, Katholieke Universiteit Leuven, Belgica, perezcol@univ-savoie.fr \\ ${ }^{2}$ Departamento de electrotecnica - ESAT, Katholieke Universiteit Leuven, Belgica, Robert.Puers@esat.kuleuven.be \\ ${ }^{3}$ Departamento de electrotecnica - ESAT, Katholieke Universiteit Leuven, Belgica, bvolckaerts@cochlear.be
}

\section{ABSTRACT}

The Cochlear Implant is broadly worn by people with deep hearing damage. This device makes up an electrode array to electrically stimulate the auditory nerves. When the electrode is implanted into the inner ear by surgery, the scala tympani is ill-treated due to the strong pressure applied on the internal ear structures. To minimize this intra-cochlear trauma, it is proposed to fabricate a micro pressure-sensor and built it in the electrode array, in such a way that the pressure applied by the electrode is measured. This work selected the MEMS SU-8 Fabry-Perot interferometer-based pressure sensor. This paper describes the sensor fabrication process carried out, and explains how to integrate this sensor with the electrode array.

Index Terms: Cochlear implant, MEMS sensor, SU8 material, Intra-cochlear trauma

\section{RESUMEN}

El implante coclear es ampliamente utilizado por personas con año auditivo profundo. Este dispositivo se compone de un conjunto de electrodos que estimulan eléctricamente los nervios auditivos. Cuando el electrodo es insertado quirúrgicamente en el oído interno, la escala timpani es maltratada debido a las considerables presiones aplicadas a las estructuras internas del oído. Esto ocasiona trauma, lo cual es indeseado. y es principalmente causado por el contacto del electrodo con las paredes de la cóclea. Para minimizar este trauma, se propone fabricar un MEMS sensor de presión para que sea integrado al conjunto de electrodos; permitiendo medir la presión aplicada al oído. Este trabajo ha seleccionado el SU8 MEMS sensor de presión basado en el efecto de interferencia óptica. Este artículo describe el proceso de fabricación del sensor y como se puede integrar al array de electrodos.

Palabras clave: implante coclear, material SU8, sensores MEMS, trauma Intra - coclear 


\section{IntRODUCTION}

The cochlear implant uses an array of electrodes to electrically stimulate the auditory nerves. Specifically, this electrode is inserted into the inner ear, nearest the modiolus. During the surgery, doctors must not damage the delicates intracochlear structures [1], [2]. However, electrode displacement along the scale tympani causes severe pressure on the inner ear walls. At present, The Cochlear Corporation commercializes two cochlear implants devices: The Nucleus 24 Contour Advanced and The Hybrid Cochlear Implant. In particular, The Hybrid implant stimulates the auditory nerves both acoustically (at low frequencies) and electrically (at high frequencies), and therefore it requires to remain the ear structures.

Both, The Nucleous 24 Contour Advanced and The Hybrid Cochlear Implant, are inserted following a technique called "Advanced Off Style (AOS)“. The aim of AOS is simply to re- duce intra-cochlear damage caused by pressure applied on cochlea walls. At present, The AOS technique has showen good results in terms of minimizing pressure prompt that a strict surgery procedure is followed.

The SU8 Fabry-Perot Interferometer-based MEMS pressure sensor is selected to be integrated with the electrode array, intented to measure the pressure during the insertion. The literature indicates that this micro-sensor is already fabricated [3],[4]. However, this sensor is made only to measure orthogonal pressure. In this application, it is prefereably the sensor readout pressure from different directions (not only orthogonal). For that, it is proposed to deposit a rubber-elastic material (polydimethyl- siloxane - PDMS) on top of the sensor membrane.

In this paper, the section II explores the AOS technique to find potentials causes of trauma during electrode insertion. The section III specifies aspects of sensor design, such as type of material and MEMS structure. The section IV introduces the SU8 Fabry-Perot interferometer- based sensor with mathematical formulations. This section also indicates the dimensions of MEMS cavity. The section $\mathrm{V}$ describes the main properties of sensor materials as well as fabrication process. At the end, the MEMS device was fabricated without depositing the PDMS material.

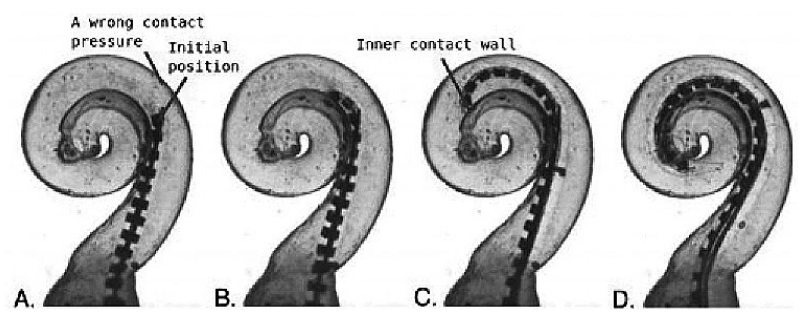

Fig. 1 AOS technique. (A) Initially, the electrode tip is placed at the beginning of the first turn. (B-D) The electrode is advanced off without contacting the lateral wall of the scala tympani. The pre-curled electrode allows closer contact with the auditory nerves. [Adapted from JRRD, Volumen 45, Number 5, 2008]

\section{Potential Intra- Cochlear Trauma}

The Cochlear Nucleus 24 Contour Advance is one of the most novel cochlear implant launched on the market. Unlike its previous models, The Nucleus 24 brings new insertion technique "Advanced Off Stylet (AOS)", and the electrode array comes up pre-curled with a soft-tip. Theoretically, the electrode allows minizing intracochlear damage while it is hosted nearest the modiolus [5]. The process of insertion begins setting the electrode over the stylet. Then, doctors put the tip of the styled mounted electrode at the beginning of cochlea first turn (see figure 1-A). A white line marked on the shank silicon enables to guide the initial position of the electrode.

The electrode array advances off without strongly touching of the outer walls. When the array is finally hosted, the stylet is removed [6], [7]. In theory, the AOS protects the walls of scale tympani due to the electrode touches softly the cochlea. 
However, the AOS is not easy to follow. According to this technique, the styled mounted electrode must be putted, initially, on the position indicated in figure 1-A. Although the white mark on the shank provides visual help to doctors for initial placement, this is not reliable due to the distance from cochleostomy (opening to access the scale tympani) to the first turn varies [8]. Incorrect initial position is often and doctors introduce the electrode array further than estimated initial position, causing trauma (see wrong contact pressure figure $1-A)$.

On the other hand, the figure 1-C shows the electrode tip touching the cochlea innerwall. In particular, the auditory nerves are connected with the organ de corti and this is located along the innerwall. Although it is not reported, strong contact pressure on the inner walls might happen with AOS and it would damage the nerve fibers. Hypothetically, this damage might cause variation of conductivity between the electrode and stimulated nerve.

The AOS technique has another disadvantage. While introducing the electrode, the inner wall experiments friction. The array has a side where the electrodes and the silicon are not equally leveled (see figure 2) and this might lead to severe friction on wall surfaces.

Around the cochlea apex, the remaining hair cells are acoustically stimulated by The Hybrid Cochlear Implant. During electrode insertion, it is a concern to preserve the whole hair cells. In the cochlea, the cells stay on the basiliar membrane, specifically on the scala media. It is feasible that during insertion, the electrode shakes strongly the perilymph (fluid in the cochlea) and this might causes strong deviation of the basilar membrane. It has been demonstrated that considerable basilar membrane displacement leads to destroy hair cells.

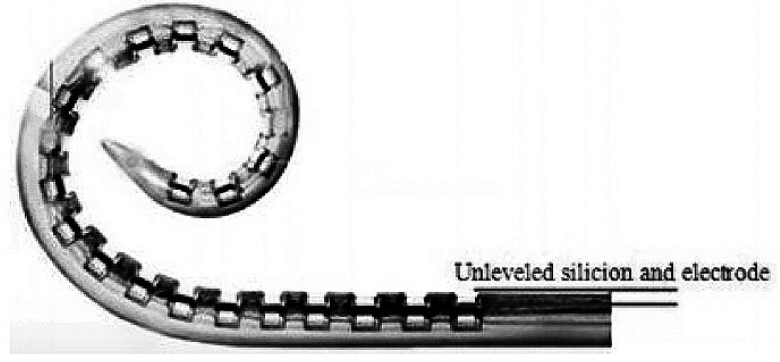

Fig. 2 The Nucleous Cochlear Implant, electrode ar-ray.Electrode and sicilion are unleveled [Adapted from www.cochlear.com]

\section{Sensor Approach}

\section{A. Applicability}

This document proposes an approach to minimize risk of intracochlear damage. A micro- pressure sensor joined at the electrode tip would measure and monitor pressure during electrode insertion. Likely, the monitoring pres- sure would plot peaks of pressure, meaninig strong contact on the cochlea walls.

\section{B. Design Aspects}

Requirements: After full insertion, the sensor must be removed from the implant. The sen sor must be highly sensitive.

The SU8 Fabry-Perot Interferometer based pressure sensor is fabricated by defining a MEMS cavity which connects with an optical fiber (see figure 3-B). For better explaining, this paper calls the cavity as "head" and the optical fiber as "tail".

Following, this paper proposes a manner of building the sensor in the implant. The silicon- electrode should have a hole all along the shank. Plus, the sensor head should be joined with the electrode tip (see figure 3-AB). The tail is only withdrawed from the electrode after full insertion.The optical fiber is removed by pulling down from one electrode end. In this way, the cavity breaks up and the sensor tail can be easily removed (see figure 3-C). 


\section{The Su8 Fabry-Perot Inter- Ferometer Based Mems Pressure Sensor}

A Fabry-Perot interferometer-based MEMS sensor is made up a source of light, guided by an optical fiber, and beamed towards a MEMS membrane (see figure 4). In particular, this membrane is made of a reflective material. When the light is transmited, most of it is sent back via the optical fiber. The power of reflective light is modulated by membrane de- flection (the amount of reflected light depends on the gap distance between the optical fiber end and the membrane, figure 4-B). A light metter is connected with one end of the optical fiber in order to measure the light back power. The relationship between light back power and membrane deflection establishes the pressure applied on the sensor.
To fabricate the micro-sensor, it is selected the polymer SU8 (see section 4 for more details). The MEMS sensor cavity is defined as shown in figure 6. In particular, the SU8 is a photo-resistor used to shape MEMS layers and it is transparent. The Fabry-Perot interferometer-based sensor requires a reflective layer, therefore, it is deposited a thin layer of Alluminum on membrane backside (see figure 4-C).

This paper proposes to apply a rubber-elastic material polydimethylsiloxane - PDMS) on top of the mbrane (see figure 4-C) to measure preasures in all irections. In this paper, the PDMS layer is only proposed and not applied to the fabricated sensor.

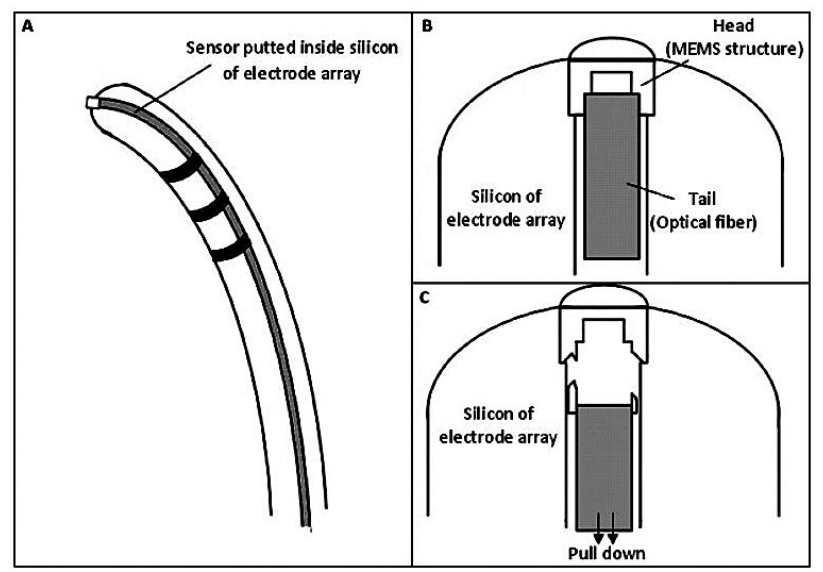

Fig. 3 Withdrawing the optical fiber from the electrode. (A-B) The sensor head sensor is fixed at the electrode tip. (C) The sensor tail is taken off by pulling down.

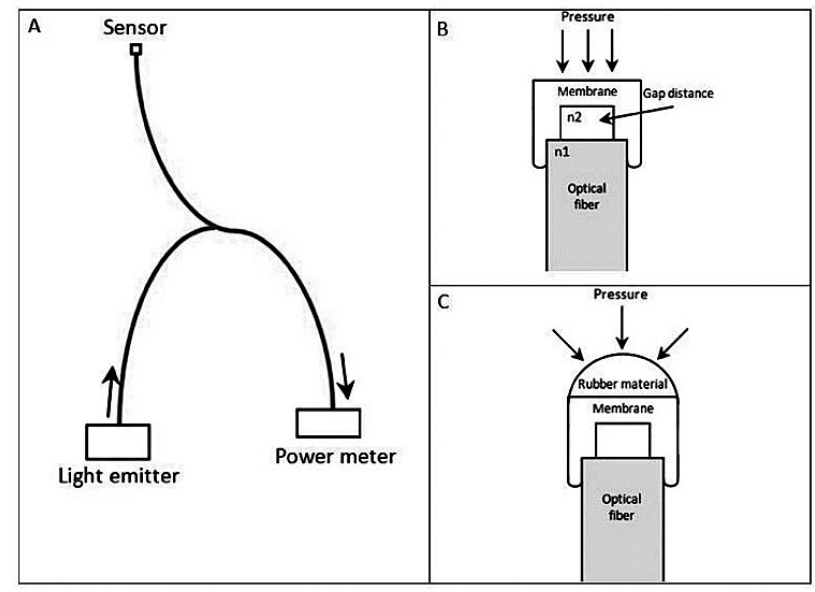

Fig. 4 SU8 Fabry-Perot interferometer-based MEMS pressure sensor. (A) The sensor with the light emmiter and power meter. (B) The SU8 Fabry-Perot sensor is coupled with the optical fiber. (C) The sensor with applied rubber-elastic material. 
TABLE I

Physical Arameters

\begin{tabular}{|l|r|}
\hline Refraction index $n 1$ & 1 \\
\hline Refraction index $n 2$ & 1.4 \\
\hline Light wavelength $(\mathrm{nm}) \lambda$ & 1310 \\
\hline Reflective backside material $r$ & 0.92 \\
\hline
\end{tabular}

\section{A. Gap Distance}

Reflected power light depends on the gap distance between the membrane and the optical fiber end. Mathematically, the effect of power light variations due to a reflective plane was studied by Yeh [8]. In the formula 1, Yeh assumed coherent light emitted orthogonally to membrane deviation is upper than $\lambda / 4$ wards a plane in motion. The SU8 MEMS pressure ensor is based on Yehs formula.

When pressure is applied on the membrane, the gap distance changes. This behavior is similar to Yehs formulation.

$$
R=\frac{r_{01}+r_{12} e^{j 2 \Omega}}{1+r_{01} r_{12} e^{j 2 \Omega}}
$$

Formula 1 refers to light-power reflection. r01 and $r 12$ are calculated by the refraction index of both the gap distance (named n2) and the optical fiber (named n1), respectively. In addition, $r$ is the reflectivity of the backside (thin layer of aluminum) and $d$ is the gap distance (see formulas 2, 3 and 4).

$$
\begin{aligned}
& r_{01}=\frac{n 1-n 2}{n 1+n 2} \\
& r_{12}=-\sqrt{r} \\
& \Omega=\frac{2 \pi \cdot n 2 . d}{\lambda}
\end{aligned}
$$

The sensor is designed at high sensitivity. The gap distance between optical fiber end and membrane needs to be calculated. For that, the formula 1 was 4.

The gap distance is selected in the middle of the wavelength maximum and minimum values.

Finally, MEMS cavity is fabricated with a gap distance of $(0.529 \mu \mathrm{m})$. It is important to guarantee this separation in order to have a sensor with predefined mathematical formulations.

\section{B. Membrane Tickness}

Membrane thickness is dimensioned such that the maximum deviation is $\lambda / 4$ [3]. The membrane is deflected till its elasticity bounding. The formula 5 calculates this deviation:

$W_{\max }=\frac{3 \cdot P_{\max } \cdot R \cdot\left(1-v^{2}\right)}{16 \cdot E \cdot t^{3}}$

Pmax is the maximum applied pressure, $R$ ishalf of membrane diameter, $v$ is the Poisson's ratio of SU8 material, $E$ is the Youngs modulus, $t$ is the thickness and Wmax is the maximum deviation.

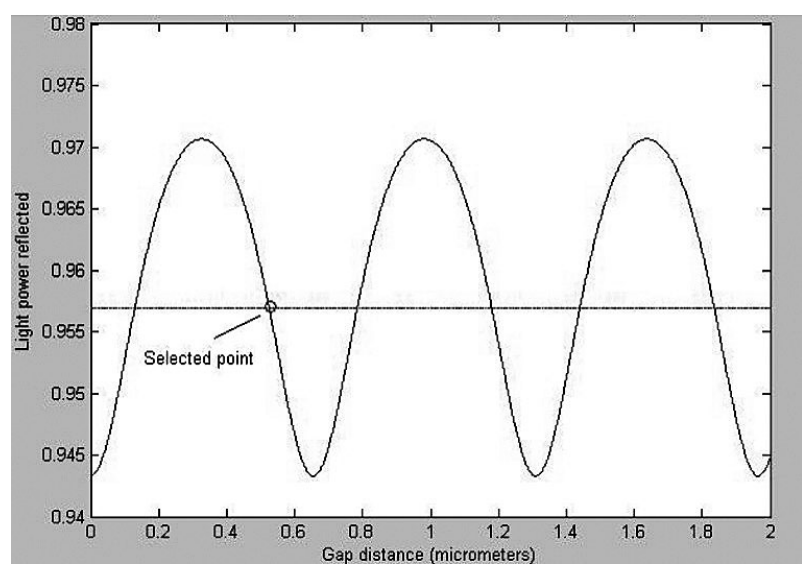

Fig. 5 Periodicity of the reflected light.

A long iterative procedure was carried out to calculate the membrane thickness, using the formula 5. However, a method explained in [3].

The sensor is designed at high sensitivity. The gap distance between optical fiber end and membrane needs to be calculated. For that, the 
formula 1 was plotted, taking the values indicated in table 1.

From figure 5, it is shown that reflected light has a periodic waveform. This behavior leads to ambiguous measurements in case of allows quickly to find the value. Due to this procedure is nonlinear, its recommended to cut the membrane deviation at $3 \lambda / 20$.

Due to membrane displacement is small, nonlinearity does not care while calcuating tickness. By using the formula 5, taking the values indicated in table 2. the membrane thickness for the sensor is $0.71 \mu \mathrm{m}$.

TABLE II

Materials Parameters

\begin{tabular}{|l|r|}
\hline Poisson's ratio & 0.22 \\
\hline Maximum pressure (units: $\mathrm{Pa})$ & 3600 \\
\hline Membrane diameter $(\mu \mathrm{m})$ & 135 \\
\hline Young's modulus $(\mathrm{GPa})$ & 2 \\
\hline
\end{tabular}

The thickness was found in this way:

- First, calculate membrane deviation by using the formula 5 and taking the values of table 2. A software tool is recommended to use.

- Iteratively, find the thickness till deviation of $\lambda / 4$ is raised. During calculation, you can modify the thickness in steps of $\lambda / 2 n 3$.

\section{Materials and Fabrication}

\section{A. The polymer SU8}

The SU8 is a negative photo-resistor based on epoxy components, widely used to create thin layer of $0.6 \mu \mathrm{m}$ to $300 \mu \mathrm{m}$. Also, it is used to fabricate multilayer coating thickness up to $2-3 \mathrm{~mm}$ [10], [11]. Chemically, it is composed by triarylium sulphonium salts and a photoacid [12], [13]. When the SU8 is irradiated with UV light, a high concentration of strong acid is formed which acts as a catalyst for the crosslinking process. When the polymer is heating, it actives the crosslinking and the sensitivity is increased. In particular, the photoacid is sensitive to UV irradiation in the range of $350-400 \mathrm{~nm}$. A normal SU8 process is described in [15].

Mechanical and chemical stability of SU8 leads to use this material to fabricate MEMS structures. Normally, the SU8 has a Youngs modulus of 2 - 5 GPa [17]. In addition, the cost of SU8 fabrication is less than silicon wafer and this makes attractive for the industry [14]. Good chemical compatibility and biostability has been also reported [16]. Unfortunately, the SU8 presents adhesion selectivity with some materials.

\section{B. The elastic Polydimethylsiloxane (PDMS)}

The polydimethylsiloxane (PDMS) is a siliconrubber based on organic polymer. This is phys-ically stable and used in MEMS as spring ma- terial, micromechanical sensors (accelerometers tactile sensors), in packing to isolate Bio micro- fluid system from external environment [19], in valves, pumps, circuits and optical systems (adaptive lenses) [20].

PDMS properties remain stable at temperature range of -50 to $200 \mathrm{C}$ [21]. Chemical and physical characteristics of PDMS are flexible (shear modulus varies between $100 \mathrm{kPa}$ and $3 \mathrm{MPa}$ ), it is optically transparent to wavelength near $256 \mathrm{~nm}$, high dielectric strength (14 $\mathrm{V} \mu \mathrm{m}-1)$, small refraction index 1.4, high permeability and compressibility with nontoxic nature [22]. Unfortunately, low surface energy does not lead to good adhesion with metals and special techniques need to be used for bonding [23]. Fabrication of PDMS layer is realized by spinning, with thickness depending on speed and time. Typical spin speed vs. thickness curve shows a hyperbolic behavior. During coating process, the rotational movements of the rubberelastic material causes shear variations between particles, and PDMS viscosity could be altered [24].

The PDMS has been also reported as biocompatible [25]. Low price and bioproperties are interesting for applications on biomedical devices 
such as catheters, drainage tubing, insulation o peacemakers and ear and nose implants [26].

\section{MEMS Cavity (sensor head)}

The sensor cavity is fabricated by creating 4 layers deposited on a crystal wafer. The firts three layers have the same diameter $(135 \mu \mathrm{m})$ but different thickness. The layer 1 is the membrane and has a thickness of $0.71 \mu \mathrm{m}$. The layer 2 is made of aluminum and it is developed on top of the layer 1 , with a thickness of $0.4 \mu \mathrm{m}$. The layer 3 defines the gap distance between the optical fiber and the membrane backside. It has a thickness of $0.57 \mu \mathrm{m}$. The last layer is made for fitting the optical fiber with the cavity. It has a thickness of $10 \mu \mathrm{m}$.

The SU8 material was acquired by The MichoChem Company [27]. The cavity fabrication process was performed in a clean room. The structure of the sensor cavity and thicknesses are shown in figure 6 .

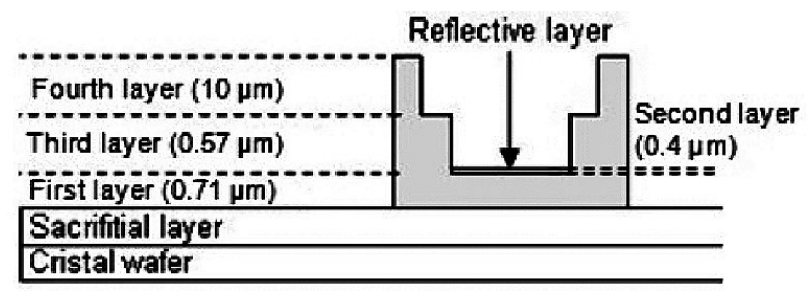

Fig. 6 The MEMS sensor cavity was fabricated by planar process (layer by layer).

\section{1) Fabrication process}

Each layer of the sensor cavity was fabricated as follows:

Initially, a sacrificial layer (Omnicoat) was deposited on a wafer, spinning at $2000 \mathrm{rpm}$ for

20 seconds. Then, it was dried on a hotplate by

$65 \mathrm{C}$ for 1 minute.

Layer 1: The SU8-2000.5 material was de- posited on the sacrifitial layer at $3 \mathrm{ml}$ on the wafer and spinning at $1100 \mathrm{rpm}$ by 20 seconds. Thickness of $0.73 \mu \mathrm{m}$ was obtained.
Layer 2: The material was Aluminum. A plasma sputter was used for this deposition. The wafer was entered in the sputter by 2.12 minutes at 300 watts. A layer thickness of 0.4

$\mu \mathrm{m}$ was obtained. The circular shape of the reflective layer was developed by lithography.

Layers 3 - 4: The layers 3 and 4 were deposited by coating the wafer again with SU8-2000.5 and SU8-2010, respectively. The fabrication process was the same as made for the first layer, unlike the spin speed was 2000 rpm (for the layer 3) and $600 \mathrm{rpm}$ (for the layer).

4). The thicknesses were $0.61 \mu \mathrm{m}$ and $11 \mu \mathrm{m}$, respectively.

\section{Optical Fiber and Cavity}

One optical fiber end is inserted in the cavity and then glued. Ideally, the optical fiber should fits exactly with the cavity hole but it is not possible at all.

A special machine was adapted to insert the optical fiber end with the cavity. By experiments, it was found that the glue must be highly viscose. Additionally, it is preferably that the optical fiber end fits almost exactly with the cavity. If the fiber remains looses after it is in, the glue can enter in the cavity and the gap distance between the membrane and optical fiber end will be glued, which is completely undesired.

\section{E. Test and Results}

The sensor was finally fabricated without depositing the PDMS material. The noglued optical fiber end was connected with a source of light and a derivation was connected with a light power meter. Several measurements was performed. The range of pressure applied was $0-3.5$ $\mathrm{KPa}$. The entire measurements were recorded and plotted using Matlab. The figure 7 shows the plotted results. 


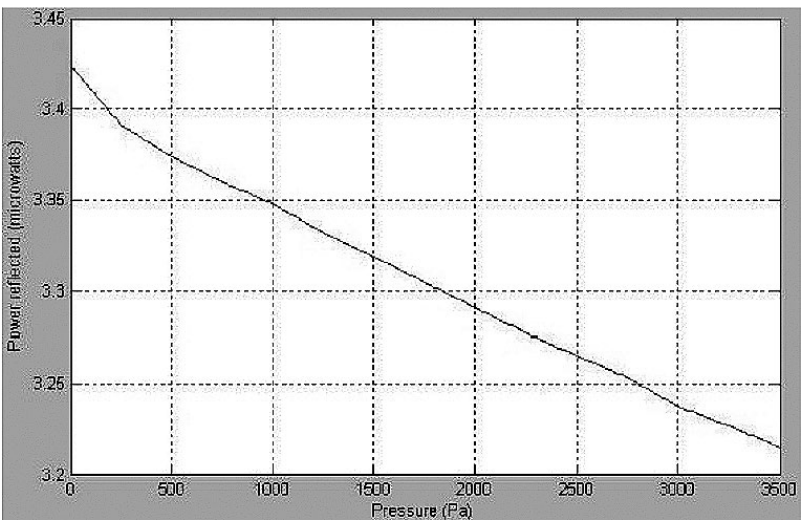

Fig. 7 The graph "reflected-light-power" vs "pressure". The measurements were performed by putting the sensor in a chamber, and varying the pressure from $0-3.5 \mathrm{KPa}$. The sensor sensitivity was $-4 \times 10-5 \mu \mathrm{W} / \mathrm{Pa}$.

\section{ConcLusions}

The next step will be to perform tests, using the electrode array with the sensor. Likely, some aspects should take into account. The cochlea is filled by a liquid and during the insertion turbulence can happens, leading to noisy measurements. This noise would not allow monitoring, properly, pressure by contact.

During fabrication process, it was found that MEMS technology still requires to overcome some limitations. MEMS assembly is not easy and for the SU8 MEMS sensor, it was very hard to join the cavity with the optical fiber. In the near future, it will require new assembly machines for this new technology.

Intra-cochlear trauma is a concern and there is not a clear way of minimize it yet. Integrating a MEMS pressure sensor into the electrode array could, in certain way, to monitor pressure during insertion but it does not garantee that traume will be minimized. New surgical technique should be evaluated.

\section{REFERENCES}

[1] O. Adunka and J. Kiefer. Impact of electrode insertion depth on intracochlear trauma. Journal: Elsevier Inc. Otoralyngology-Head and Neck Surgery. United States: pags 374-382, 2006.

[2] P. Roland and W. Gstonttner and O. Adunka. Method for hearing preservation in cochlear implant surgery, Journal: Elsevier Inc. United States: pags 93 - 100, 2005.

[3] F. Ceyssens and M. Driesen and R. Puers. An optical absolute pressure sensor for high-temperature applications, fabricated directly on a fiber, Journal: Micromechanics and Microengi- neering. Belgium, 2005.

[4] G. Hill and R. Melamud and F. Declerq and A. Davenpoirt and I. Chan and H and B. Pruit SU-8 MEMS Fabry- Perot pressure sensor, Journal: micromechanics and micro- engineering. Vol 13. Pags 52-62 United States, 2007.

[5] B. Chen and G. Clark and R. Jones. Evaluation of trayectories and contacts pressures for the straight nucleous cochlear implant electrode array - a two dimensional application of finite element analysis, Elsevier Inc. United states, 2002.

[6] J. Roland. A model for cochlear implant insertion and force evaluation: Results with new electrode design and insertion technique, New york University: Laryngoscope. Pag 115. United States, 2005.

[7] T. Ran Hsu. MEMS and Microsystems: Design and Manufac- ture, McGraw-Hill: Science/Engineering/ Math. 1st Ed. 2001.

[8] MEMSCYCLOPEDIA, SU8 resist. Thick photo-resistor for MEMS. http://memscyclopedia.org/.

[9] J. Hammacker and A. Fuelle and J. Flaemig and J. Saupe and B. Loechele and J. Grim Stress engineering and me- chanical propierties of SU-8 layers for mechanical applications, Microsyst Tech United states, 14:1515-1523. 2008.

[10] P. Abgrall and V. Conedera and H. Camon and A. Gue and N. Nguyen SU-8 as a structural material for labson- chips and microelectromechanical systems. Electrophoresis 28. 2007.

[11] F. Lindemans and N.F. de Rooij Microsystems technologies for implantable applications. J. Micromech and Microeng 17. R50-R80.2007.

[12] J. Lotters and W. Olthuis and P.H. Veltink and P. Bergveld The mechanical propierties of the rubber elasctic polymer poly- dimethylsiloxane for sensor applications. Journal: Microchem and Microeng. Pags 145-147. 1997.

[13] A. del Campo and C. Greiner Su-8: a prhotoresistor for high aspect ratio and 3D submicron lithography. Journal: Mi- crochem and Microeng. 2007.

[14] N. J. Shirtcliffe and S. Aquil and C. Evans and G. McHale and M.I. Newton and C. Perry and P. Roach The use of high aspect ratio photoresistor (SU-8) for super-hydrophobic pattern prototyping. Journal: Microchem and Microeng. Vol14. Pags 1384-1389. 2004. 